\title{
Development of an improved vaccine for anthrax
}

\author{
Stephen H. Leppla, ${ }^{1}$ John B. Robbins, ${ }^{2}$ Rachel Schneerson, ${ }^{2}$ and Joseph Shiloach ${ }^{3}$ \\ ${ }^{1}$ National Institute of Dental and Craniofacial Research, \\ ${ }^{2}$ National Institute of Child Health and Human Development, and \\ ${ }^{3}$ National Institute of Diabetes and Digestive and Kidney Diseases, NIH, Bethesda, Maryland, USA \\ Address correspondence to: Stephen Leppla, Oral Infection and Immunity Branch, NIDCR, Building 30, Room 303, \\ 30 Convent Drive, Bethesda, Maryland 20892-4350, USA. Phone: (301) 594-2865; Fax: (301) 402-0396; E-mail: Leppla@nih.gov.
}

J. Clin. Invest. 109:141-144 (2002). doi:10.1172/JCI200216204.

Bacillus anthracis are aerobic or facultatively anaerobic Gram-positive, nonmotile rods measuring $1.0 \mu \mathrm{m}$ wide by $3.0-5.0 \mu \mathrm{m}$ long. Under adverse conditions, $B$. anthracis form highly resistant endospores (Figure 1). These are found in soil at sites where infected animals previously died. Interest in the pathogenesis, immunity, and vaccine development for anthrax was heightened by the deliberate contamination of mail with $B$. anthracis spores soon after the September 11 attacks. At that time, the only US-licensed human vaccine (anthrax vaccine adsorbed, or AVA) was not available because the manufacturer, BioPort Corp., had not received FDA certification of its new manufacturing process.

Although Pasteur had already demonstrated protection of sheep by injection of heat-attenuated $B$. anthracis cultures in 1881, our current knowledge of immunity to anthrax in humans remains limited. Widespread vaccination of domesticated animals with attenuated strains such as the Sterne strain began in the 1930s and has virtually abolished anthrax in industrialized countries. In the US, the licensed human vaccine (AVA, newly renamed BioThrax) is an aluminum hydroxide-adsorbed, formalin-treated culture supernatant of a toxigenic, noncapsulated, nonproteolytic $B$. anthracis strain, V770-NP1-R, derived from the Sterne strain (1). AVA was developed in the early 1950s, when purified components of $B$. anthracis were not available. Its only demonstrable protective component is the protective antigen (PA) protein (2). A similar culture supernatant-derived human vaccine is produced in the United Kingdom.

Data from a 1950s trial of wool-sorters immunized with a vaccine similar to AVA, coupled with long experience with AVA and the United Kingdom vaccine, have shown that a critical level of serum antibodies to the $B$. anthracis PA confers immunity to anthrax (3, 4). As early as 1959, a British Ministry of Labour report noted that, following the introduction of regular immunization the previous year, the staff of the Government Wool Disinfection Station in Liverpool were free of the disease "despite the high risk to which they are exposed" (5). AVA also protects laboratory animals and cattle from both cutaneous and inhalational challenge with $B$. anthracis $(1,6,7)$. Although safe and efficacious (8), AVA has limitations that justify the widespread interest in developing improved vaccines consisting solely of well-characterized components. First, standardization of AVA is based on the manufacturing process and a potency assay involving protection of guinea pigs challenged intracutaneously with $B$. anthracis spores $(7,9)$. PA is not measured in the vaccine, and there is no standardized assay of PA antibodies in animals or humans vaccinated with AVA. These factors probably explain why it has been difficult to maintain consistency of AVA. Second, this vaccine contains other cellular elements that probably contribute to the relatively high rate of local and systemic reactions (8). Finally, the schedule of AVA administration (subcutaneous injections at 0,2 , and 4 weeks and 6,12, and 18 months with subsequent yearly boosters) is probably not optimal. This schedule, introduced in the 1950s, was designed for rapid induction of immunity (10), but it was recently shown that increasing the interval between the first two injections enhances the level of AVA-induced antibodies to PA (11). Moreover, there is no experimental support for including the injections given at 6,12 , and 18 months.

\section{B. anthracis as a human pathogen}

Humans contract anthrax almost exclusively from contact with, ingestion of, or inhalation of $B$. anthracis spores. Cutaneous anthrax results from a break in the skin and has a mortality rate of about $20 \%$ in untreated cases. Incubation is usually $2-3$ days, although it can occur within 12 hours and as late as 2 weeks. A small papule appears, followed by a surrounding ring of vesicles about 24 hours later. The lesions ulcerate and become black and edematous. In pulmonary anthrax, inhaled spores are carried by macrophages from the lungs to adjacent lymph nodes. The spores germinate, multiply, and cause septicemia. Primary inflammation of the lungs (pneumonia) may not be detectable. Because the signs and symptoms of infection are mimicked by 


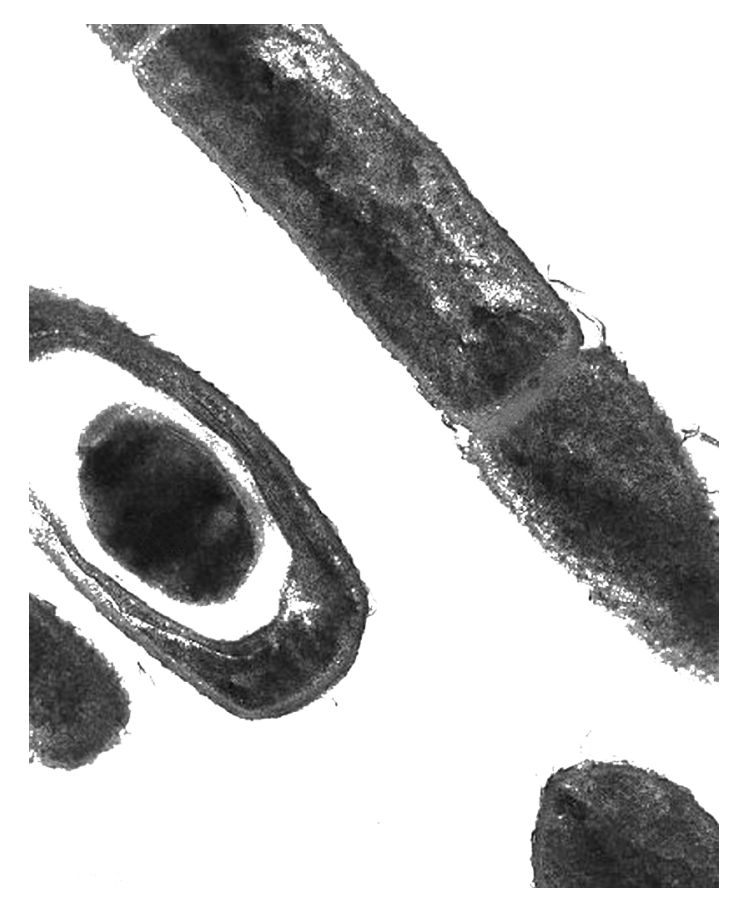

Figure 1

A spore (left) and vegetative cells and a chain of vegetative rod cells of $B$. anthracis. Electron micrograph courtesy of the Centers for Disease Control and Prevention.

administration of anthrax toxin to monkeys, anthrax can be considered a toxin-mediated disease.

The virulence of $B$. anthracis results from the action of materials that are expressed from genes on two large plasmids, pX01 and pX02 $(12,13)$. pX01 encodes the proteins that make up the anthrax toxin. The massive edema and organ failure seen in anthrax patients are caused largely by the action of three individually nontoxic proteins: protective antigen $(\mathrm{PA}, 83 \mathrm{kDa})$, edema factor (EF, adenylate cyclase, $89 \mathrm{kDa}$ ), and lethal factor (LF, zinc protease, $90 \mathrm{kDa}$ ) (14). The latter two combine with the PA to form edema toxin and lethal toxin, respectively. PA, EF, and LF fit the A-B toxin model proposed by Gill (15). Thus, following its interaction with host cells, PA (the " $\mathrm{B}$ " subunit) is activated by the cellular protease furin, causing the release of a $20-\mathrm{kDa}$ $\mathrm{N}$-terminal domain (16). The remaining $63-\mathrm{kDa}$ polypeptide creates a heptameric structure that constitutes a channel in the host cell membrane (17) through which LF and EF (each of which represents an alternative " $\mathrm{A}$ " subunit in this model) are translocated to the cytosol. The unregulated adenylate cyclase activity of EF leads to production of unphysiologically high concentrations of cAMP, one consequence of which is incapacitation of phagocytic cells (14). LF cleaves several mitogen-activated protein kinase kinases, thereby blocking signal transduction pathways by which host immune cells normally respond to pathogens $(18,19)$.

Plasmid pX02 encodes the poly- $\gamma$-linked D-glutamic acid (PGA) capsule, demonstrable by a Quellung (antibody-induced swelling) reaction (20). Strains lacking pX02 are avirulent. PGA confers virulence to
B. anthracis by its antiphagocytic activity, in the same way that capsular polysaccharides confer virulence to the pneumococci.

\section{The essential role of anti-toxin antibodies in immunity to $B$. anthracis}

Experience with AVA and PA (native or recombinant) in animals challenged by inhalation or by intracutaneous injection is extensive and points strongly to PA as an essential component of living, inactivated, or proteinbased vaccines for anthrax. Thus, strains of $B$. anthracis cured of pX01, and therefore lacking the ability to express the components of anthrax toxin, are not virulent and do not confer immunity to animals (21) (with the exception of mice, as discussed below). Moreover, PA IgG antibodies, either actively induced or passively administered as polyclonal or monoclonal proteins, confer protection to mice, rabbits, rats, guinea pigs, and monkeys challenged with $B$. anthracis either intracutaneously or by aerosol. Pitt et al. (22) recently reported an excellent correlation between the level of AVA-induced antibodies and immunity to inhalational anthrax in rabbits. Conversely, antibodies to EF or LF alone do not confer strong immunity to infection, although antibodies to LF induced by a DNA vaccine protect against toxin challenge (23) and merit further study for their potential to act synergistically with antibodies to PA.

In certain other bacterial infections, antitoxins (serum-neutralizing antibodies) can exert antibacterial activity. For example, the toxin-mediated respiratory diseases caused by Corynebacterium diphtheriae and Bordetella pertussis are prevented by immunization with their respective toxoids (24). Both pathogens are almost always confined to the epithelial surface of the respiratory tract, where their secreted toxins cause local inflammation and inactivate phagocytic cells. Vaccineinduced antitoxins confer immunity to these diseases by permitting phagocytosis of C. diphtheria and B. pertussis. In the case of anthrax, there is the additional effect that antibodies protect mononuclear cells from toxin action, preventing the release of intracellular cytokines, lymphokines, and other agents that may contribute to the fever, shock, and multiple organ failure that characterize anthrax (25). The antibacterial effect of vaccine-induced antitoxin, therefore, is indirect and is mediated by protection of lymphoid cells against the actions of anthrax toxin, thus permitting phagocytosis and killing of $B$. anthracis.

Toxin-neutralizing mouse mAb's identify two critical sites on PA (Figure 2); a site on domain 4 that binds the cellular receptor, and a site on the PA 63-kDa heptamer to which the LF and EF bind (26). It is probable that vaccine-induced immunity depends on antibodies to these two sites, and possibly to other sites. The key role of the receptor-binding site is shown by the ability of domain 4 alone to induce antibodies that protect mice from infection with $B$. anthracis (27), and by the potent toxin-neutralizing ability of affinity-enhanced recombinant antibodies directed to an epitope that includes amino acids 680-692 within domain 4 (28). 


\section{The potential role of antibodies}

\section{to capsular polypeptide, spores, and LF}

PGA is a poor immunogen, probably because of its resistance to proteolysis in antigen-presenting cells and its simple, repeating structure, which makes it a $\mathrm{T}$ cell-independent antigen (29). Thus, there has not yet been a definitive test of whether anti-capsular antibodies contribute to immunity to $B$. anthracis. The species of animals used to evaluate PGA is critical. Encapsulated $B$. anthracis strains are quite virulent in mice, regardless of whether they produce toxin (21). PGA, therefore, rather than the toxin, is the major virulence factor in mice, and vaccines based on PA show reduced efficacy in this species (30). For this reason, it may be easier to demonstrate a role for anti-PGA antibodies in protective immunity using mice, once effective strategies for inducing such antibodies have been established. However, it will be difficult to extrapolate conclusions about capsular antibodies to human anthrax infection, given that the contributions of PGA and PA to pathogenesis differ substantially between mice and humans.

Unexpectedly, antibodies to PA have been found to have sporicidal properties in vivo. PA antibodies both suppress germination and enhance phagocytosis of spores by macrophages (31). Active immunization of guinea pigs and mice with formalin-inactivated spores has also been reported to confer immunity to infection $(30,32)$. Studies showing that antibodies to LF can neutralize toxin in vivo were noted above (23). However, these studies on other candidate immunogens lack information about the specificity and concentration of the antibodies mediating the protection observed.
Evidence that other antigens can contribute to immunity suggests that the most effective vaccines would contain multiple antigens. Immunization trials with these additional antigens, individually and in combination, could lead to highly efficacious thirdgeneration vaccines. However, in the short term, improved anthrax vaccines will consist primarily of PA. Thus, the National Institute of Allergy and Infectious Diseases has an accelerated program for vaccine development that seeks to make 25 million doses of a recombinant $P A$ vaccine available within two years (33).

\section{How will an investigational anthrax vaccine be standardized?}

Limited clinical data with AVA and substantial animal experimentation indicate that a critical level of serum anti-PA antibodies confer immunity to both cutaneous and inhalational anthrax $(8,34)$. An improved anthrax vaccine, therefore, could be a single-component, purified protein that elicits concentrations of PA antibodies comparable to those induced by AVA. However, there are a number of uncertainties that may complicate the seemingly simple transition to a recombinant PA vaccine. First, it is unclear what concentration of serum PA antibodies in humans confers immunity to anthrax. Data for the efficacy of AVA are limited to one trial and long experience without a vaccine failure. For this reason, it is not obvious what point in the AVA injection schedule, or what resulting level of protective anti-PA antibodies, should be chosen as the standard for comparison with new vaccine candidates. Second, the level of Ab's required to protect people from the effects of a bioterrorist attack is uncertain, because the number of spores inhaled under those circumstances might greatly

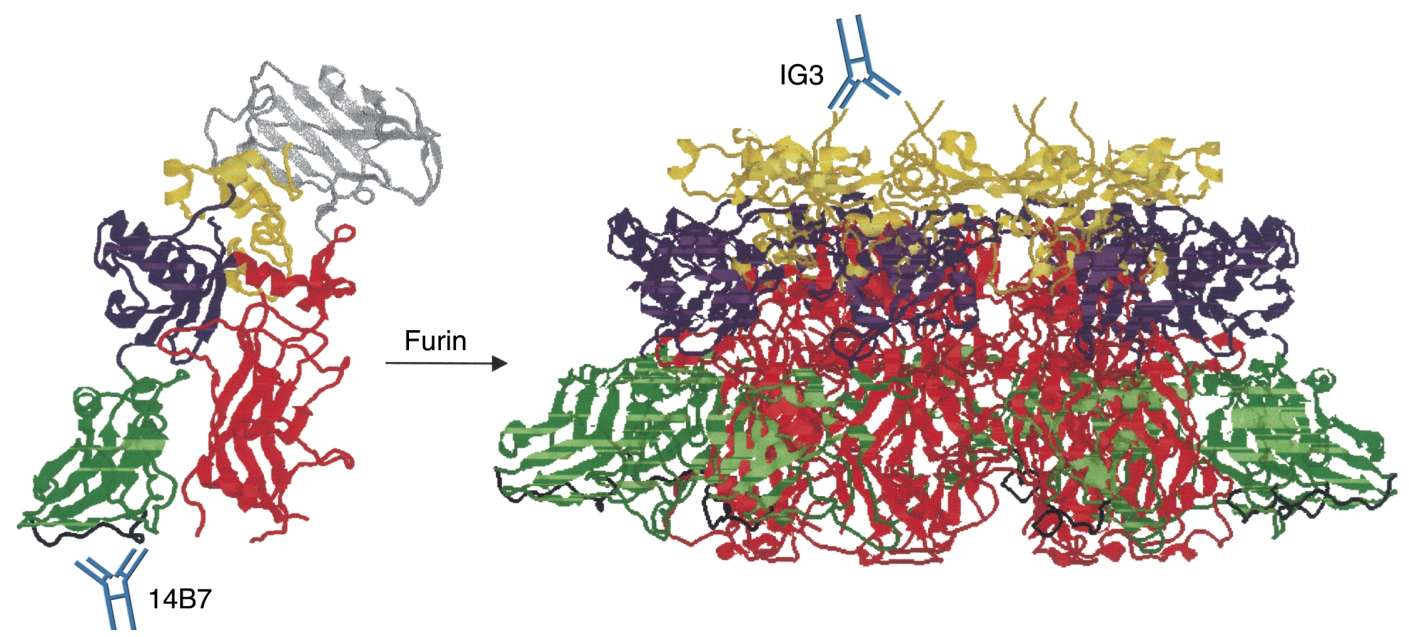

Figure 2

PA regions targeted by neutralizing antibodies. PA monomer (left) is cleaved by cellular furin, releasing domain 1a (amino acids 1-167, colored gray), and allowing the remaining domains to assemble into a heptameric channel (right). Domains remaining in the heptamer are $1 \mathrm{~b}$ (yellow), 2 (red), 3 (blue), and 4 (green). Indicated in black within domain 4 is the "small loop," amino acids 680-692, which is involved in binding to the PA receptor. The neutralizing mouse mAb 14B7 reacts with this region, preventing PA binding to the receptor. In the heptamer, the "top" of domain 1a contains a surface on which LF and EF bind to initiate internalization. Neutralizing mouse mAb $1 \mathrm{G} 3$ binds to this same surface, competing with EF and LF, and thereby blocking their action. This figure was created with Protein Explorer (35), available at http://www.umass.edu/microbio/chime/explorer, using existing PA monomer and heptamer structure files contained in an Atlas of Macromolecules available at http://www.umass.edu/ microbio/chime/explorer/index2.htm. 
exceed that encountered by the previously studied population of wool-sorters. Would a vaccine need to protect against $5 \mathrm{LD}_{50}$ or $5000 \mathrm{LD}_{50}$ ? (We presume that exposure would be to aerosolized spores from an "anthrax bomb," but it is possible that $B$. anthracis could be added to drinking water or food. This latter route would not be as immediately dangerous, but the spores would not be totally inactivated by boiling and could pose a continual threat). Third, selection of appropriate doses and schedules for pediatric vaccination also requires study. Would the schedule used for primary immunization with the diphtheria and tetanus toxoids be satisfactory? Finally, several methodological concerns need to be addressed in designing and testing new vaccines. Would animal experiments showing that LF, PGA, or spore antigens provide enhanced protection compared with PA alone justify the addition of these components to a future vaccine? Can physicochemical and immunochemical assays accurately predict the efficacy of a recombinant vaccine? Answers to these questions are not out of reach, and it is probable that recombinant vaccines with reduced reactogenicity, a shorter immunization schedule, and equal or greater protective efficacy relative to AVA will be available soon.

1. Puziss, M., et al. 1963. Large-scale production of protective antigen of Bacillus anthracis in anaerobic cultures. Appl. Microbiol. 11:330-334.

2. Turnbull, P.C., Broster, M.G., Carman, J.A., Manchee, R.J., and Melling, J. 1986. Development of antibodies to protective antigen and lethal factor components of anthrax toxin in humans and guinea pigs and their relevance to protective immunity. Infect. Immun. 52:356-363.

3. Brachman, P.S., et al. 1962. Field evaluation of a human anthrax vaccine. Am. J. Public Health. 52:632-645.

4. Food and Drug Administration. 1985. Biological products: bacterial vaccines and toxoids; implementation of efficacy review. Proposed rule. Federal Register. 50:51002-51117.

5. 1959. Report of the Committee of Inquiry on Anthrax. Ministry of Labour (United Kingdom). Her Majesty's Stationery Office. London, United Kingdom. 83 pp.

6. Wright, G.G., Green, T.W., and Kanode, R.G. 1954. Studies on immunity in anthrax. V. Immunizing activity of alum-precipitated protective antigen. J. Immunol. 73:387-391.

7. Fellows, P.F., et al. 2001. Efficacy of a human anthrax vaccine in guinea pigs, rabbits, and rhesus macaques against challenge by Bacillus anthracis isolates of diverse geographical origin. Vaccine. 19:3241-3247.

8. 2002. The anthrax vaccine. Is it safe? Does it work? L.M. Joellenbeck, L.L. Zwanziger, J.S. Durch, and B.L. Strom, editors. National Academy Press. Washington, DC, USA. 265 pp. Online at www.nap.edu.

9. 1994. Anthrax Vaccine Adsorbed (AVA). Code of Federal Regulations. 21. Parts 600-799, chapter 1(4-1-94), subpart C.

10. Puziss, M., and Wright, G.G. 1963. Studies on immunity in anthrax. X. Gel-adsorbed protective antigen for immunization of man. J. Bacteriol. 85:230-236.

11. Pittman, P.R., et al. 2000. Anthrax vaccine: increasing intervals between the first two doses enhances antibody response in humans. Vaccine. 19:213-216.

12. Green, B.D., Battisti, L., Koehler, T.M., Thorne, C.B., and Ivins, B.E. 1985. Demonstration of a capsule plasmid in Bacillus anthracis. Infect. Immun. 49:291-297.

13. Uchida, I., Hashimoto, K., and Terakado, N. 1986. Virulence and immunogenicity in experimental animals of Bacillus anthracis strains harbouring or lacking $110 \mathrm{MDa}$ and $60 \mathrm{MDa}$ plasmids. J. Gen. Microbiol. 132:557-559.

14. Leppla, S.H. 1999. The bifactorial Bacillus anthracis lethal and oedema toxins. In Comprehensive sourcebook of bacterial protein toxins. J.E. Alouf and J.H Freer, editors. Academic Press. London, United Kingdom. 243-263.

15. Gill, D.M. 1978. Seven toxin peptides that cross cell membranes. In Bacterial toxins and cell membranes. J. Jeljaszewicz and T. Wadstrom, editors. Academic Press Inc. New York, New York, USA. 291-332.

16. Klimpel, K.R., Molloy, S.S., Thomas, G., and Leppla, S.H. 1992. Anthrax toxin protective antigen is activated by a cell surface protease with the sequence specificity and catalytic properties of furin. Proc. Natl. Acad. Sci. USA. 89:10277-10281.

17. Singh, Y., Klimpel, K.R., Goel, S., Swain, P.K., and Leppla, S.H. 1999. Oligomerization of anthrax toxin protective antigen and binding of lethal factor during endocytic uptake into mammalian cells. Infect. Immun. 67:1853-1859.

18. Duesbery, N.S., et al. 1998. Proteolytic inactivation of MAP-kinasekinase by anthrax lethal factor. Science. 280:734-737.

19. Erwin, J.L., et al. 2001. Macrophage-derived cell lines do not express proinflammatory cytokines after exposure to Bacillus anthracis lethal toxin. Infect. Immun. 69:1175-1177.

20. Makino, S., Uchida, I., Terakado, N., Sasakawa, C., and Yoshikawa, M. 1989. Molecular characterization and protein analysis of the cap region, which is essential for encapsulation in Bacillus anthracis. J. Bacteriol. 171:722-730.

21. Welkos, S.L., Vietri, N.J., and Gibbs, P.H. 1993. Non-toxigenic derivatives of the Ames strain of Bacillus anthracis are fully virulent for mice: role of plasmid pX02 and chromosome in strain-dependent virulence. Microb. Pathog. 14:381-388.

22. Pitt, M.L., et al. 2001. In vitro correlate of immunity in a rabbit model of inhalational anthrax. Vaccine. 19:4768-4773.

23. Price, B.M., et al. 2001. Protection against anthrax lethal toxin challenge by genetic immunization with a plasmid encoding the lethal factor protein. Infect. Immun. 69:4509-4515.

24. Schneerson, R., Robbins, J.B., Taranger, J., Lagergard, T., and Trollfors, B. 1996. A toxoid vaccine for pertussis as well as diphtheria? Lessons to be relearned. Lancet. 348:1289-1292.

25. Hanna, P.C., Acosta, D., and Collier, R.J. 1993. On the role of macrophages in anthrax. Proc. Natl. Acad. Sci. USA. 90:10198-10201.

26. Little, S.F., et al. 1996. Characterization of lethal factor binding and cell receptor binding domains of protective antigen of Bacillus anthracis using monoclonal antibodies. Microbiology. 142:707-715.

27. Flick-Smith, H.C., et al. 2002. A recombinant carboxy-terminal domain of the protective antigen of Bacillus anthracis protects mice against anthrax infection. Infect. Immun. 70:1653-1656.

28. Maynard, J.A., et al. 2002. Protection against anthrax toxin by recombinant antibody fragments correlates with antigen affinity. Nat. Biotechnol. 20:597-601.

29. Goodman, J.W., and Nitecki, D.E. 1966. Immunochemical studies on the poly-gamma-D-glutamyl capsule of Bacillus anthracis. I. Characterization of the polypeptide and of the specificity of its reaction with rabbit antisera. Biochemistry. 5:657-665.

30. Brossier, F., Levy, M., and Mock, M. 2002. Anthrax spores make an essential contribution to vaccine efficacy. Infect. Immun. 70:661-664.

31. Welkos, S., Little, S., Friedlander, A., Fritz, D., and Fellows, P. 2001. The role of antibodies to Bacillus anthracis and anthrax toxin components in inhibiting the early stages of infection by anthrax spores. Microbiology. 147:1677-1685

32. Cohen, S., et al. 2000. Attenuated nontoxinogenic and nonencapsulated recombinant Bacillus anthracis spore vaccines protect against anthrax. Infect. Immun. 68:4549-4558.

33. 2002. Development and testing of vaccines against anthrax. Request for proposal. National Institute of Allergy and Infectious Diseases, NIH. http://www.niaid.nih.gov/contract/archive/RFP0226.pdf.

34. Friedlander, A.M., Pittman, P.R., and Parker, G.W. 1999. Anthrax vaccine: evidence for safety and efficacy against inhalational anthrax. JAMA. 282:2104-2106.

35. Martz, E. 2002. Protein Explorer: easy yet powerful macromolecular visualization. Trends Biochem. Sci. 27:107-109. 